\title{
Research Square \\ Estimating the critical points of an epidemic: An application of non-epidemiological logistic growth model for COVID-19
}

Ivan Bezerra Allaman ( $\sim$ iballaman@uesc.br)

State University of Santa Cruz https://orcid.org/0000-0003-0883-0466

Enio Galinkin Jelihovschi

State University of Santa Cruz https://orcid.org/0000-0002-7286-1198

\section{Research Article}

Keywords: point of asymptote, point of acceleration, point of decel- eration, inflection point, accumulated cases, virus

Posted Date: October 15th, 2020

DOI: https://doi.org/10.21203/rs.3.rs-93146/v1

License: (c) (1) This work is licensed under a Creative Commons Attribution 4.0 International License.

Read Full License

Version of Record: A version of this preprint was published at Brazilian Journal of Biometrics on June 10th, 2022. See the published version at https://doi.org/10.28951/bjb.v40i2.576. 


\title{
Estimating the critical points of an epidemic: An application of non-epidemiological logistic growth model for COVID-19
}

\author{
Ivan Bezerra Allaman ${ }^{1, *}$ and Enio Galinkin Jelihovschi ${ }^{1,+}$ \\ ${ }^{1}$ State University of Santa Cruz, Department of exact and technological sciences, Ilhéus/BA, 45662-900, Brazil \\ *iballaman@uesc.br \\ +these authors contributed equally to this work
}

\begin{abstract}
Epidemiological models have become a very important tool in understanding an epidemic development, mainly because they help researchers in finding good and new strategies in their fight against its spread. Several models have been proposed up to now, some are mathematical others apply models from other areas. The SIR and SEIR among others, mainly focus on the variable response and on epidemiological parameters as the basic reproduction number $\left(\mathrm{R}_{0}\right)$ and infection rate per unit of time, nevertheless they do not focus on the variable 'time'. We propose the use of the variable time using the logistic model as it is generally used to describe the growth of animals. This model is important because it allows the estimation of the points of acceleration and deceleration, the point of maximum growth and the asymptotic point of the epidemic. This is only possible when the epidemic curve is stable and has an ' $S$ ' shape. In this work we use the variable 'accumulated cases' of China and Italy and point out the main socioeconomic facts that occurred in each period of the estimated critical points from the logistic growth model.
\end{abstract}

\section{Introduction}

The first official statement by the World Health Organization (WHO) on COVID-19 pandemic acted as a trigger on the scientific community to start a real effort to study and publish papers in order to provide a way of understanding what has been happening and therefore help and guide the fight against the virus. In this way, new mathematical models like Sales ${ }^{1}$ and Shaikh ${ }^{2}$ added to the well-known epidemiological models SIR, SEIR, Generalized Logistic Model, Generalized Richards Model and Generalized Growth Model.

The logistic models, among the mathematical models proposed for epidemics, stand out because they present an ' $\mathrm{S}$ ' format and, therefore, make it possible to obtain interesting information about the curve such as absolute and relative growth rate, points of acceleration and asymptote whose parameters may have biological interpretation, see Seber ${ }^{3}$. All logistic models proposed in the literature up to now are those derived from the Richards model.

In the case of epidemics, Chowell ${ }^{4}$ used, in addition to the generalized growth model and the generalized Richards model, the logistic model to characterize the contagiousness, forecast patterns and final burden of Zika Epidemics. However, the authors were vague about the logistic model used. On the other hand, $\mathrm{Wu}^{5}$, put it explicitly the parameters of the logistic model used to describe and characterize an epidemic, nonetheless the authors expressed the logistic model as a solution of differential equations, as it is commonly used in epidemiological models. Only Zhou ${ }^{6}$ presented the logistic growth model in the form of a function which is the closest to the model proposed in this work.

All models proposed so far studied the dynamics of virus transmission given some specific scenario, none of these studies presented a proposal to determine when acceleration or deceleration occur in the number of individuals infected or when the number of those infected will stabilize. Time is a very important factor. We do not try to predict the future given predetermined settings, we start from an already established scenario, and then adjust a logistic model in order to estimate the critical points of this model and thereafter try to understand how political, social or environmental factors are related to such critical points of the proposed curve.

Our proposal is to place the variable time as the main factor using the classic logistic model. Derived from Richards model, this model is commonly used to describe the growth of animals ${ }^{7}$. In order to avoid any confusion with the logistical models used in epidemiological studies also derived from the Richards model, we will call our model a 'non-epidemiological logistic growth model'.

In short, the aim of our work is to estimate critical points of the non-epidemiological logistical growth such as the point of maximum acceleration, inflection point, point of maximum deceleration and asymptotic deceleration point using the number of 
accumulated cases as the dependent variable. Furthermore, we use the main stock exchange indexes and some main political facts in China and Italy to show possible relationships to the critical points of the curve. China and Italy were chosen due to the fact that they have shown a steady epidemic curve by the time of our study.

\section{Results}

The values of the arima model for each country are shown in table 1 .

Table 2 shows the point estimates and confidence intervals of parameters and critical points. The point of map has been estimated at 34.93 days for China and 50.92 days for Italy counting from the first official case reported for both countries. The estimated value of the point of ip was 41.68 and 65.53 days for China and Italy respectively. For the point of $\mathbf{d m p}$, the estimates were 48.43 days for China and 80.14 days for Italy. Finally, the point estimated of adp for China and Italy were 57 and 94 days respectively.

Figure 2 places the stock exchange index and the percentage of deaths during the period studied with each period established by the estimated critical points through the logistic model for both China and Italy.

We highlight below the main interventions in each period comprising the critical points of the logistic model for China.

1. Period from the beginning of the pandemic to the map point (35 days).

(a) The Huanan Seafood Wholesale Market in Wuhan city was closed for environmental sanitation and disinfection ${ }^{8}$;

(b) Beginning of the Chinese festival ${ }^{9}$;

(c) Prohibition of Wuhan residents to leave the city and closing off the public transport in Wuhan and Hubei province ${ }^{10}$;

2. The facts mentioned between the period of the point of map and the point of ip ( 7 days), were taken from Times ${ }^{11}$.

(a) Wuhan began to classify patients and adopted centralized patient management, dedicated hospital built to fight against COVID-19;

(b) Huoshenshan makeshift hospital was put into use;

(c) The first 3 make shift hospitals were put into use;

3. A single fact stands out between period of the points of ip and mdp (6 days)

(a) Following a chance in the diagnosis criteria of confirmed cases, the Chinese mainland reported 15, 152 new confirmed COVID-19 cases, including 13,332 in Hubei Province.

4. Among the points of mdp and adp period (9 days), the following facts stand out :

(a) The number of newly discharged patients began to surpass the new confirmed case;

(b) Provinces and regions across the Chinese mainland began to downgrade the COVID-19 emergency response;

(c) The China-WHO joint expert team held a press conference in Beijing;

5. Finally, from point of adp until 2020-06-30 (126 days), the following facts stand out:

(a) New local cases in the Chinese mainland declined to single digits;

(b) Hubei Province gradually revoked the outbound travel restrictions;

(c) National Health Comission began to daily report the asymptomatic cases;

(d) All inbound travelers to China were mandated to undergo a compulsory nucleic acid test;

(e) Leading group of the CPC (Central Commitee for Novel Coronavirus Prevention and Control published guidelines for expediting work resumption with strict adherence to epidemic control;

(f) Wuhan lifted outbound travel restrictions; 
(g) Beijing and its nearby provinces downgraded the emergency response to the second-level;

(h) Hubei Province downgraded its emergency response to the second-level;

(i) The State Council released Guidelines on conducting COVID-19 prevention and control on an ongoing basis;

The Dow Jones Shangai, which is the main index of the Chinese stock exchange, has shown a drop until to the point of map (figure 2). Then, the index rose until shortly after the point of adp, the stabilization point of the number of people infected, then a sharp drop for 27 days and a resumption of growth until the last evaluation day.

In Italy, the main facts in the periods as shown in figure 2, were selected from the wikipedia website ${ }^{12}$.

1. From the beginning until the point of map (51 days), the following facts stand out:

(a) The government announced a new decree imposing the quarantine of more than 50,000 people from 11 municipalities in Northern Italy;

(b) Schools were closed in ten municipalities in Lombardy, one in Veneto and one in Emilia Romagna;

(c) All sporting events in the regions of Lombardy and Veneto, which included three Serie A football matches in those regions, as well as one in Piedmont, that were to be played the following day;

(d) The Ministry of Health announced that it had engaged 31 laboratories in Italy to carry out the analysis of swabs from suspected COVID-19 cases;

(e) Multiple regions in Italy such as Liguria, Trentino Alto Adige, Friuli Venezia Giulia, Abruzzo and Marche decided to close all schools and universities for two days to a week;

(f) The Italian government imposed the shutdown of all schools and universities nationwide for two weeks as the country reached 100 deaths from the outbreak;

(g) The government announced that all sporting events in Italy would be cancelled until at least 3 April, but the ban does not include Italian clubs or national teams participating in international competitions;

(h) The Ministry of Health ordered tighter regulations on free movement;

(i) Conte announced further restrictions within the nationwide lockdown, by halting all non-essential production, industries and businesses in Italy, following the rise in the number of new cases and deaths in the previous days;

2. Between the point of map and point of ip (15 days) the following facts stand out:

(a) The government extended the period of lockdown until 13 April, with health minister Speranza saying that the restrictive measures had begun to yield the first positive results;

3. Among the point of ip and point of mdp points (14 days), the following facts stand out:

(a) A government's decree closed all Italian ports until 31 July, stating that they do not ensure the necessary requirements for the classification and definition of 'safe place', established by the Hamburg Rules on maritime search and rescue;

(b) Conte announced the prolongation of the lockdown until 3 May, as well as the reopening of some businesses like bookshops and forestry;

4. Between the point of $\mathbf{m d p}$ and the point of adp (13 days), a single fact stands out:

(a) The Prime Minister announced a starter plan for the so-called 'phase 2', that would start from 4 May;

5. Finally, from the point of adp until 2020-06-30 (59 days), the following facts stand out:

(a) Conte announced the government plan for the easing of restrictions - Starting from 18 May most businesses could reopen, and free movement was granted to all citizens within their Region;

(b) Movement across Regions was still banned for non-essential motives;

The main index of the Italian stock exchange (FTSE MIB) dropped until a few days before the first critical point of map, then remained stable until the last day of our study. 


\section{Discussion}

The critical points estimated through the classic non-epidemiological logistic model are the only ones which support the researcher to speak in terms of 'acceleration' and 'asymptote' of a given epidemic. Although many studies mention the terms transmission speed and 'curve stabilization', none of them make in a suitable statistical way.

The model hitherto proposed, was intended to describe the outbreak of the epidemic, that is, how the variables such as number of infected people, number of people recovered should behave as a function of epidemiological parameters. Some researchers applied new models, as in the case of Sales ${ }^{1}$, who makes an application of the prey-predator model proposed by Loka and Volterra for COVID-19, and Giordano ${ }^{13}$ that uses the model of SIDARTHE to discriminate between detected and undetected cases of infection and between different severity of illness (SOI), non-life-threatening cases (asymptomatic and paucisymptomatic; minor and moderate infection) and potentially life threatening cases (major and extreme) that require ICU admission. Shaikh ${ }^{2}$, proposed a mathematical model using fractional derivative. Established epidemiological models were also applied like SIR ${ }^{14-16}$, SEIR ${ }^{6,17,18}$, Generalized Logistic Model $^{5}$, Generalized Richards Model ${ }^{5}$ and Generalized Growth Model $^{5}$. Our approach is unique because it estimates when (time) the main critical points of an epidemic occur using a simple model, that is, without differential equations that requires deep mathematical skills for the user. The model proposed in this paper allows us to answer questions like: 'How many days are necessary to stop the epidemic?', ${ }^{19}$. In China, it lasted 57 days until the point of adp, in Italy, 93 days.

Some other questions may arise and any researcher interested in the field could use the tool proposed above to try to answer them. For instance, why the time between the begining of the outbreak and the point of map in China was shorter (35 days) than in Italy (51 days)? As already pointed out by other studies, the lockdown was indeed an effective measure in the battle against the spread of an epidemic ${ }^{10}$. In China, the lockdown was implemented 23 days, in Wuhan, epicenter of the pandemic at that time, after the first case officially reported. In Italy, the lockdown was implemented 37 days after the first occurrence, in Lombardy together with 14 additional northern and central provinces like Piedmont, Emilia-Romagna, Veneto and Marche. In addition to the lockdown, air pollution may have been another major factor in Italy as showed in Coccia ${ }^{19}$. Another interesting result was the proximity between the lockdown and the point of map. In China, this time was 12 days while in Italy it was 14 days. Does this behavior remain the same in other countries?

The logistics growth model complements all the studies already proposed for the understanding of a given epidemic, as it puts the variable time as the most important one. Our proposal has the limitation of being applied only after the epidemic has already been stabilized. On the other hand, it allows the estimation of more precise parameters that have not yet been addressed in the literature and, does not make conjectures about the future and its various scenarios as is the case with epidemiological models.

For instance, after estimating the critical points of the logistic growth model, the researchers could try to understand why the time until the peak of infection or the time until the stabilization were different among countries; what health, social and economics measures or which environmental factors may contribute to decrease or increase the time among the critical points.

\section{Methods}

\section{Data collection}

The database used in this paper is available on the website githubusercontent.

Since the data are updated daily, we considered the day 2020-06-30 as deadline. The countries included in the analyzes are China and Italy, because those countries managed to stabilize contamination by SARS COVID-19 by the time of our deadline. We must point out that the new cases obtained on 2020-02-13 (China) and 2020-06-20 (Italy) were considered very atypical compared to the values of the previous day and the day after. Thereat, these values were replaced by the average of the previous day with the day after.

As for the data on the indexes of the Chinese stock exchanges at Dow Jones in Shangai and at FTSE Mib in Italy, those indexes were obtained from the website www. investing. com. Since the stock exchange doesn't work on weekends and holidays, the indexes on these days have been replaced by the immediately preceding days so that the evaluated period of the COVID 19 cases coincided with the stock indexes.

\section{Estimation of the Autoregressive Integrated Moving Average (ARIMA) model}

As the accumulated variable (object of this study) is a consequence of the daily cases and this in turn is characterized as a temporal series whose values and residues are correlated over time, we adjusted an arima model for all countries using the auto.arima function forecast package version $8.12^{20}$. However, since this model requires an assumption of normality, homoscedasticity and independence, we made a boxcox transformation. 


\section{Estimation of the logistic growth model}

We first adjusted the arima model in order to determine the estimated accumulated values and then used them to adjust the logistic model. The reparametrization was:

$$
f(x)=\frac{A}{1+B e^{-k x}}
$$

where,

A: is the parameter that represents the model's asymptote as $x \rightarrow \infty$;

$\mathrm{B}$ : it is a parameter without biological interpretation;

$\mathrm{k}$ : is the parameter that represents the maturity index of an organism.

The parameters of the logistic model were determined using the function $n l s$ of the stats package version 4.0 .0 using the Gauss-Newton algorithm. The maximum acceleration points (map), inflection point (ip), maximum deceleration point (mdp) and asymptotic deceleration point (adp) were determined from model 1. The points of map and mdp were determined using the third derivative, the point of ip was by using the second derivative and the point of adp by using the method of non-significant difference ${ }^{21}$. The equations of each critical point are:

1. For map and mdp we first use the following equation,

$$
\frac{d^{3}}{d x^{3}}=\frac{A B k^{3} \mathrm{e}^{k x}\left(\mathrm{e}^{2 k x}-4 B \mathrm{e}^{k x}+B^{2}\right)}{\left(\mathrm{e}^{k x}+B\right)^{4}}
$$

then finding the points where the derivative is equal to zero.

$$
\operatorname{map}=\frac{\ln (2 B-\sqrt{3} B)}{k}
$$

and mdp

$$
\operatorname{mdp}=\frac{\ln (\sqrt{3} b+2 b)}{k}
$$

2. For the ip we use,

$$
\frac{d^{2}}{d x^{2}}=-\frac{A B k^{2} \mathrm{e}^{k x}\left(\mathrm{e}^{k x}-B\right)}{\left(\mathrm{e}^{k x}+B\right)^{3}}
$$

then finding the points where the derivative is equal to zero.

$$
\text { ip }=\frac{\ln (B)}{k}
$$

3. The adp is found by using the non-significant difference method. We use the algorithm shown below to explain how it works.

(a) Calculate $\Delta_{x_{i}}=p \cdot A-f\left(x_{i}\right)$ where $A$ is the estimated parameter of the logistic model asymptote, $p$ is the proportion of the asymptote, a fixed value between 0 and 1 and $f\left(x_{i}\right)$ is the value of the logistical function (eq. 1) at point $x_{i}$.

(b) Estimate the variance of $\Delta$ that is $S^{2}(\Delta)=\operatorname{diagonal}\left(f(w)^{\prime} \cdot \Sigma \cdot f(w)\right)$ where,

$$
f(w)=\left[\begin{array}{lll}
\partial \Delta / \partial A & \partial \Delta / \partial B & \partial \Delta / \partial k
\end{array}\right]^{\prime}
$$

and $\Sigma$ is the of variance-covariance matrix obtained from the estimated logistics regression model.

(c) For each $x_{i}$ find the statistic $T=\Delta_{x_{i}} / \sqrt{S^{2}\left(\Delta_{x_{i}}\right)}$.

(d) Set a level of significance $\alpha$ and evaluate the quantile $t_{\alpha}$ of the student's distribution with $n-p$ degrees of freedom where $n$ is the sample size and $p$ is the number of parameters of the model.

(e) Compare until $T_{x_{i}}<t_{\alpha}$. The value of $x_{i}$ where this occurs for the first time is the asymptote point.

Figure 1 identifies the critical points of the first and second derivative from the logistic model. The $95 \%$ confidence intervals were calculated for all parameters and critical points ${ }^{3,22}$. All analyzes were performed using the R software ${ }^{23}$. 


\section{References}

1. Sales, J. H. Epidemic covid mathematical model. Int. J. Lat. Res. Sci. Tech. 72, 1-5 (2020).

2. Shaikh, A. S., Shaikh, I. N. \& Nisar, K. S. A mathematical model of covid-19 using fractional derivative: Outbreak in india with dynamics of transmission and control. Ad. Diff. Eq. 1-19 (2020).

3. Seber, G. A. F. \& Wild, C. J. Nonlinear Regression (John Wiley \& Sons, Inc., 1989).

4. Chowell, G. et al. Using phenomenological models to characterize transmissibility and forecast patterns and final burden of zika epidemics. PLoS currents 8 (2016).

5. Wu, K., Darcet, D., Wang, Q. \& Sornette, D. Generalized logistic growth modeling of the covid-19 outbreak in 29 provinces in china and in the rest of the world. arXiv preprint arXiv:2003.05681 (2020).

6. Zhou, X. et al. Forecasting the worldwide spread of covid-19 based on logistic model and seir model. medRxiv (2020).

7. Freitas, A. D. Curvas de crescimento na produção animal. Rev. Bras. Zootec. 34, 786-795 (2005).

8. Organization, W. H. et al. Coronavirus disease (covid-2019) situation reports. 1 (2020).

9. Leung, K., Wu, J. T., Liu, D. \& Leung, G. M. First-wave covid-19 transmissibility and severity in china outside hubei after control measures, and second-wave scenario planning: a modelling impact assessment. The Lancet (2020).

10. Qiu, Y., Chen, X. \& Shi, W. Impacts of social and economic factors on the transmission of coronavirus disease 2019 (covid-19) in china. J. Pop. Econo. 1 (2020).

11. Times, G. Timeline of china's battle against covid-19 pandemic. https://www.globaltimes.cn/content/1190796.shtml (2020). Access date: 1 jun. 2020.

12. contributors, W. Covid-19 pandemic in italy — Wikipedia, the free encyclopedia (2020). [Online; accessed 21-July-2020].

13. Giordano, G. et al. Modelling the covid-19 epidemic and implementation of population-wide interventions in italy. Nat. Med. 1-6 (2020).

14. Biswas, K., Khaleque, A. \& Sen, P. Covid-19 spread: Reproduction of data and prediction using a sir model on euclidean network. arXiv preprint arXiv:2003.07063 (2020).

15. Chen, Y.-C., Lu, P.-E. \& Chang, C.-S. A time-dependent sir model for covid-19. arXiv preprint arXiv:2003.00122 (2020).

16. Weissman, G. E. et al. Locally informed simulation to predict hospital capacity needs during the covid-19 pandemic. An. intern. med. (2020).

17. Klôh, V. P. et al. The virus and socioeconomic inequality: An agent-based model to simulate and assess the impact of interventions to reduce the spread of covid-19 in rio de janeiro, brazil/o vírus e a desigualdade socioeconômica: um modelo baseado em agentes para simular e avaliar o impacto de intervenções para reduzir a disseminação do covid-19 no rio de janeiro, brasil. Braz. J. Heal. Rev. 3, 3647-3673 (2020).

18. Lai, S. et al. Effect of non-pharmaceutical interventions for containing the covid-19 outbreak in china. medRxiv DOI: 10.1101/2020.03.03.20029843 (2020). https://www.medrxiv.org/content/early/2020/03/13/2020.03.03.20029843.full.pdf.

19. Coccia, M. Factors determining the diffusion of covid-19 and suggested strategy to prevent future accelerated viral infectivity similar to covid. Sci. Tot. Environ. 138474 (2020).

20. Hyndman, R. J. \& Khandakar, Y. Automatic time series forecasting: the forecast package for R. J. Stat. Softw. 26, 1-22 (2008).

21. Mischan, M. M., Pinho, S. Z. d. \& Carvalho, L. A.-d. R. d. Determination of a point sufficiently close to the asymptote in nonlinear growth functions. Sci. Agric. 68, 109-114 (2011).

22. Mischan, M. M. \& de Pinho, S. Z. Modelos não lineares: Funções assintóticas de crescimento (2014).

23. Team, R. C. R: A Language and Environment for Statistical Computing. R Foundation for Statistical Computing, Vienna, Austria (2020).

\section{Author contributions statement}

Ivan B. Allaman conceived the idea, performed the data analysis and wrote the article. Enio G. Jeliovschi improved and corrected the wording and translated the article.

\section{Additional information}

The author(s) declare no competing interests. 


\begin{tabular}{|c|c|c|l|}
\hline $\mathrm{p}$ & $\mathrm{d}$ & $\mathrm{q}$ & Country \\
\hline 4 & 1 & 3 & China \\
1 & 2 & 2 & Italy \\
\hline
\end{tabular}

Table 1. Values of $\mathrm{p}, \mathrm{d}$ and $\mathrm{q}$ from the arima model of the daily cases for China and Italy

\begin{tabular}{|l|l|l|l|l|}
\hline Country & Parameters & Fitted & \multicolumn{2}{|l}{ Confidence interval (95\%) } \\
\cline { 4 - 5 } & & & Lower & Upper \\
\hline China & A & 73273.75 & 73048.35 & 73499.35 \\
& B & 3406.8 & 2614.1 & 4498.81 \\
& k & 0.20 & 0.19 & 0.20 \\
& amp & 34.93 & 34.69 & 35.17 \\
& ip & 41.68 & 41.52 & 41.84 \\
& dmp & 48.43 & 48.18 & 48.68 \\
& adp & 57.00 & & \\
& A & 240775.4 & 238842.12 & 242731.6 \\
& B & 368.04 & 297.6 & 460.66 \\
& k & 0.09 & 0.09 & 0.09 \\
& amp & 50.92 & 50.36 & 51.48 \\
& ip & 65.53 & 65.11 & 65.96 \\
& dmp & 80.14 & 79.43 & 80.85 \\
& adp & 94.00 & & \\
\hline
\end{tabular}

map-maximum acceleration point,mdp-maximum deceleration point,ipinflection point,adp-asymptotic deceleration point

Table 2. Critical points and $95 \%$ confidence interval estimates adjusted for China and Italy 

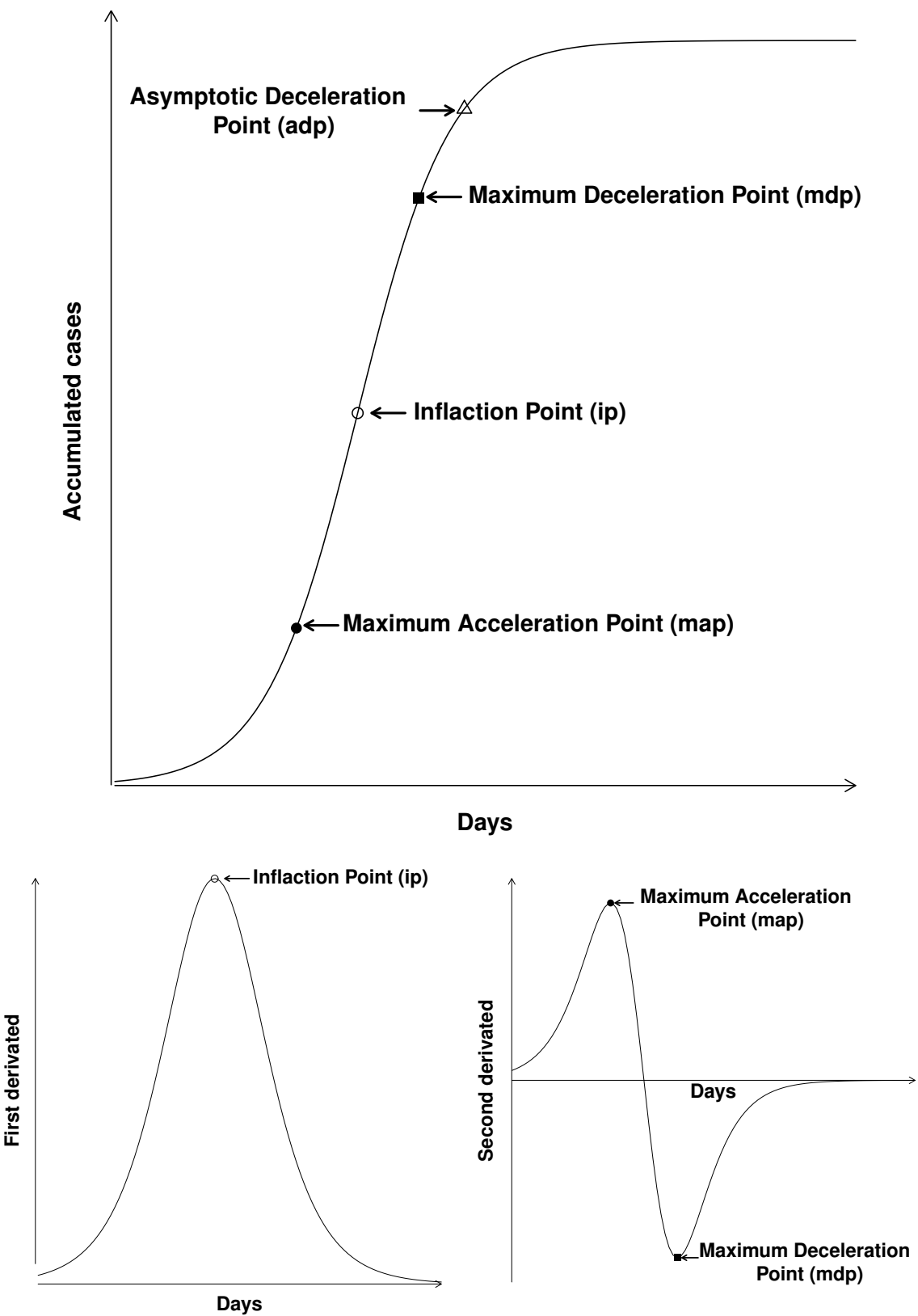

Figure 1. Curves of the logistic model, the first derivative, the second derivative and their respective critical points 

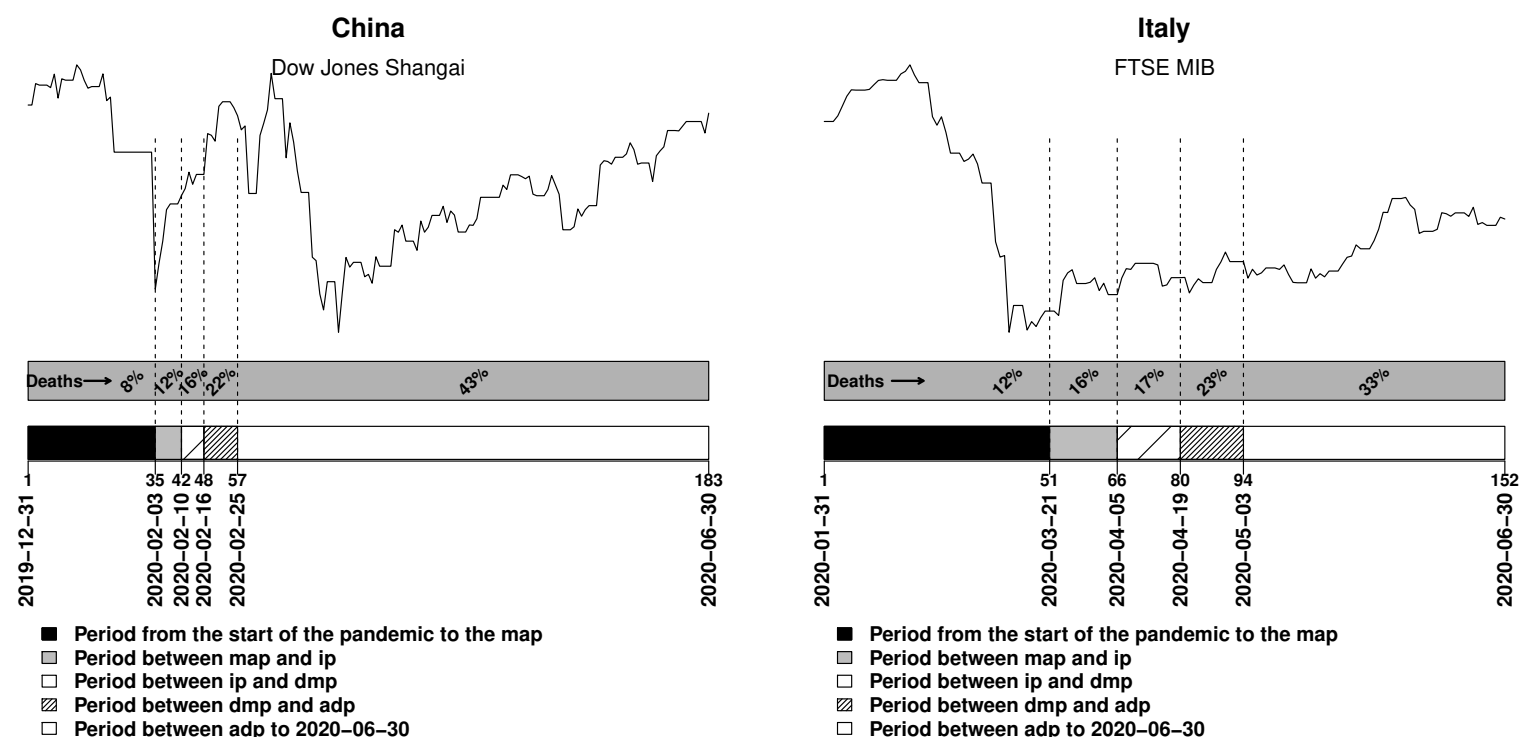

Figure 2. Stock exchange index (line graph), percentage of deaths and periods comprising the critical points of the logistic growth model: maximum acceleration point (map), inflaction point (ip), maximum deceleration point (mdp) and asymptotic deceleration point (adp) 
Figures
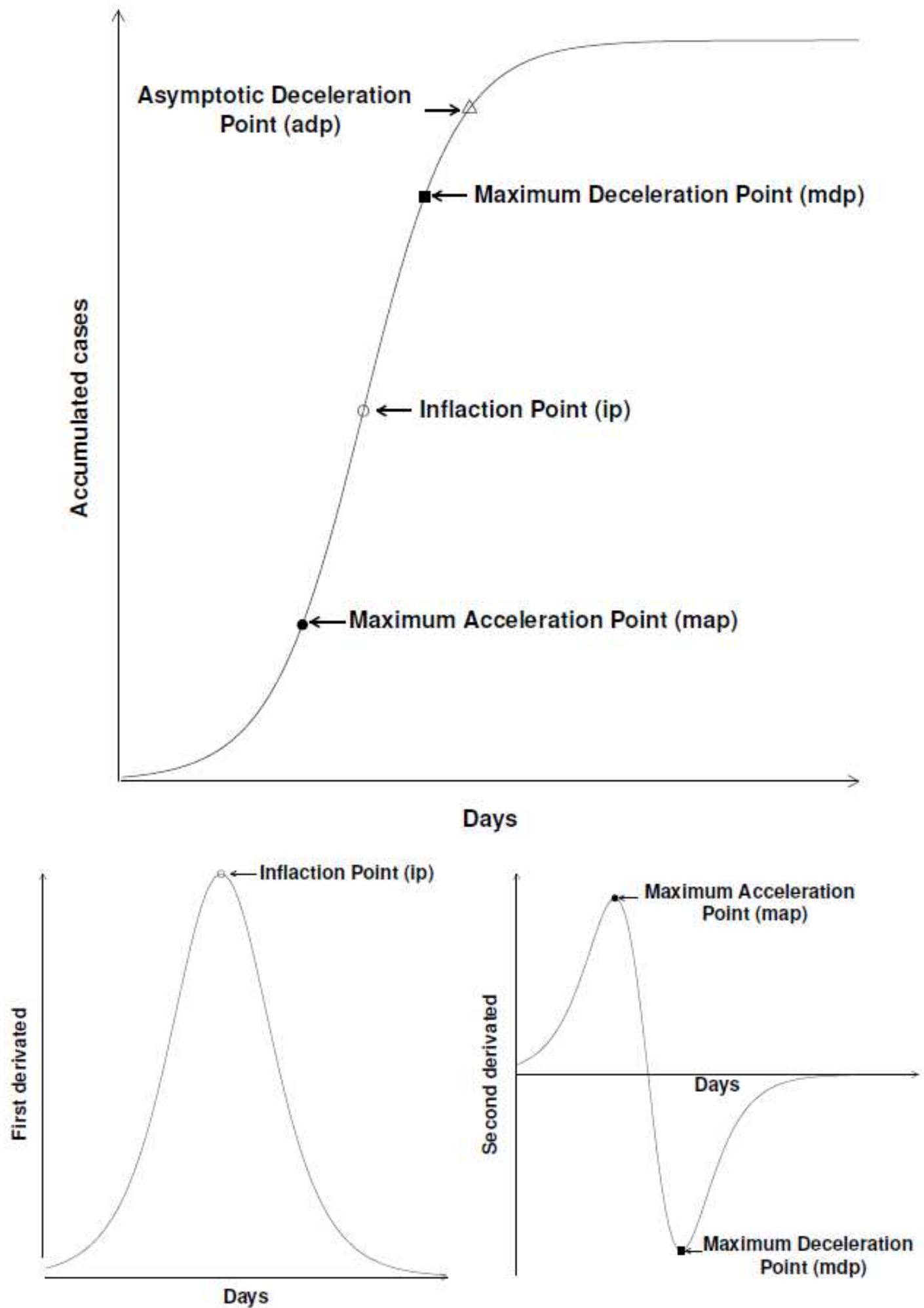

Figure 1

Curves of the logistic model, the first derivative, the second derivative and their respective critical points 
China
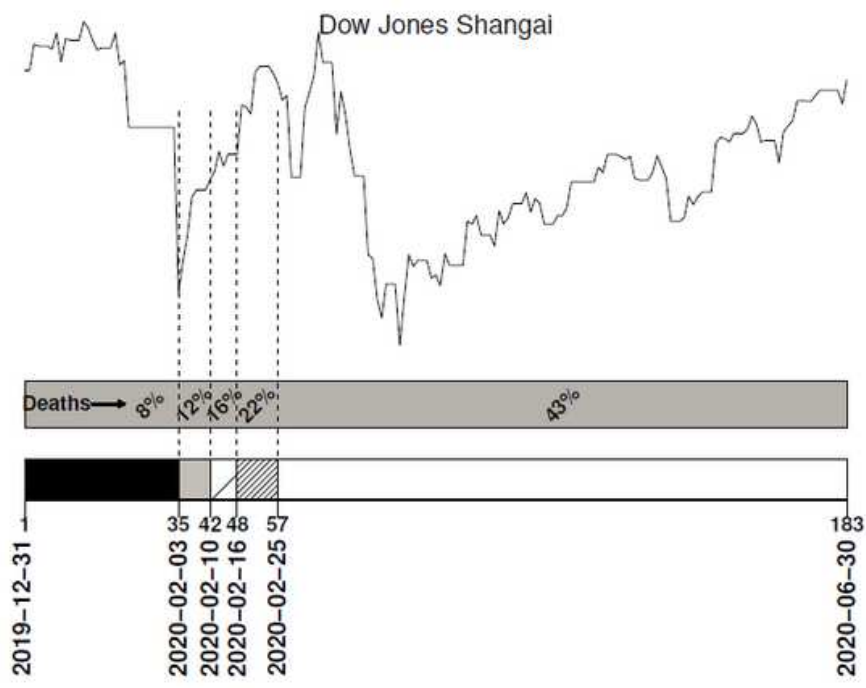

- Period from the start of the pandemic to the map

$\square$ Period between map and ip

$\square$ Period between ip and dmp

Period between dmp and adp

$\square$ Period between adp to 2020-06-30
Italy
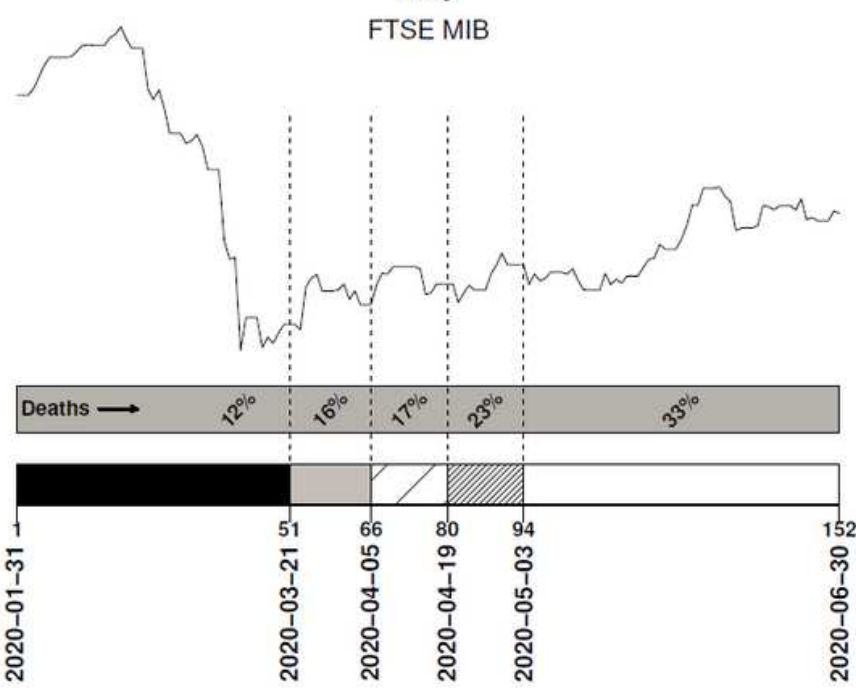

Period from the start of the pandemic to the map

Period between map and ip

Period between ip and dmp

Period between dmp and adp

Period between adp to 2020-06-30

\section{Figure 2}

Stock exchange index (line graph), percentage of deaths and periods comprising the critical points of the logistic growth model: maximum acceleration point (map), inflaction point (ip), maximum deceleration point (mdp) and asymptotic deceleration point (adp) 